\title{
SPADnet: Embedded coincidence in a smart sensor network for PET applications
}

\author{
C. Bruschini $^{\text {b,* }}$, E. Charbon ${ }^{\mathrm{a}, \mathrm{b}}$, C. Veerappan ${ }^{\mathrm{a}}$, L.H.C. Braga ${ }^{\mathrm{c}}$, N. Massari ${ }^{\mathrm{c}}$, M. Perenzoni $^{\mathrm{c}}$, \\ L. Gasparini ${ }^{\text {c }}$, D. Stoppa ${ }^{\text {c }}$, R. Walker ${ }^{\text {d }}$, A. Erdogan ${ }^{\text {d }}$, R.K. Henderson ${ }^{\text {d }}$, S. East ${ }^{\mathrm{e}}$, L. Grant ${ }^{\mathrm{e}}$, \\ B. Jatekos ${ }^{f}$, F. Ujhelyi ${ }^{\mathrm{f}}$, G. Erdei ${ }^{\mathrm{f}}$, E. Lörincz ${ }^{\mathrm{f}}$, L. André ${ }^{\mathrm{g}}$, L. Maingault $^{\mathrm{g}}$, V. Reboud ${ }^{\mathrm{g}}$, \\ L. Verger ${ }^{\mathrm{g}}$, E. Gros d'Aillon ${ }^{\mathrm{g}}$, P. Major ${ }^{\mathrm{h}}$, Z. Papp ${ }^{\mathrm{h}}$, G. Németh $^{\mathrm{h}}$ \\ a Delft University of Technology, Delft, The Netherlands \\ ${ }^{\mathrm{b}}$ Ecole Polytechnique Fédérale de Lausanne (EPFL), Lausanne, Switzerland \\ ${ }^{\mathrm{c}}$ Fondazione Bruno Kessler (FBK), Trento, Italy \\ ${ }^{\mathrm{d}}$ University of Edinburgh, Edinburgh, United Kingdom \\ e STMicroelectronics (RE'D) Ltd, Edinburgh, United Kingdom \\ ${ }^{\mathrm{f}}$ Budapest University of Technology and Economics (BME), Budapest, Hungary \\ ${ }^{g}$ CEA-LETI, Grenoble, France \\ ${ }^{\mathrm{h}}$ Mediso Medical Imaging Systems Ltd, Budapest, Hungary
}

\section{A R T I C L E I N F O}

\section{Available online 7 September 2013}

Keywords:

Digital PET

Single-Photon Avalanche Diodes (SPADs)

CMOS

Networking

TSV

\begin{abstract}
A B S T R A C T
In this paper we illustrate the core technologies at the basis of the European SPADnet project (www. spadnet.eu), and present the corresponding first results. SPADnet is aimed at a new generation of MRI-compatible, scalable large area image sensors, based on CMOS technology, that are networked to perform gamma-ray detection and coincidence to be used primarily in (Time-of-Flight) Positron Emission Tomography (PET). The project innovates in several areas of PET systems, from optical coupling to single-photon sensor architectures, from intelligent ring networks to reconstruction algorithms. In addition, SPADnet introduced the first computational model enabling study of the full chain from gamma photons to network coincidence detection through scintillation events, optical coupling, etc.
\end{abstract}

(c) 2013 Elsevier B.V. All rights reserved.

\section{Introduction}

Medical diagnostics today takes advantage in great part of image sensing and reconstruction techniques. Early detection of several diseases - cancer, in particular - has been benefitted from these techniques, thus contributing to saving lives and improving the quality of life of those affected. In this paper we present the first results from the SPADnet project (www.spadnet.eu) aimed at a new generation of MRI-compatible, scalable large area image sensors, based on CMOS technology, that are networked to perform gamma-ray detection and coincidence to be used primarily in (Time-of-Flight) Positron Emission Tomography (PET).

SPADnet innovates in several areas of PET systems, from optical coupling to single-photon sensor architectures, from intelligent ring networks to reconstruction algorithms. In addition, SPADnet introduced the first computational model enabling study of the full chain from gamma photons to network coincidence detection through scintillation events, optical coupling, etc.

\footnotetext{
* Corresponding author.Present address: EPFL, SCI STI EC, INF 135 (Bâtiment INF), Station 14, CH-1015 Lausanne, Switzerland. Tel.: +41 216933911.

E-mail address: claudio.bruschini@epfl.ch (C. Bruschini).
}

We have fabricated and tested the first version of the SPADnet photosensor, a fully digital CMOS Silicon Photomultiplier (SiPM) with $8 \times 16$ pixels individually capable of photon time stamping and energy accumulation [1]. The sensor also provides a real-time output of the total detected energy at up to 100 Msamples/s and on-chip discrimination of gamma events. These events can then be routed to the SPADnet ring network, which operates at $2 \mathrm{Gbps}$, providing real-time processing and coincidence determination within the network itself; this architecture simplifies the construction of the overall system and allows the scaling of the system to larger arrays of detectors. This may result in better and faster image reconstruction.

In the following sections we detail SPADnet's core technologies and highlight the first experimental results from the SPADnet sensor, along with the main project achievements in optimization of scintillation coupling, fill factor recovery through nano-imprinted optical concentrators, Gbps network, and reconstruction issues.

\section{SPADnet core technologies and results}

In the SPADnet approach, a number - typically a few tens of natively digital, intelligent SPAD (Single-Photon Avalanche 


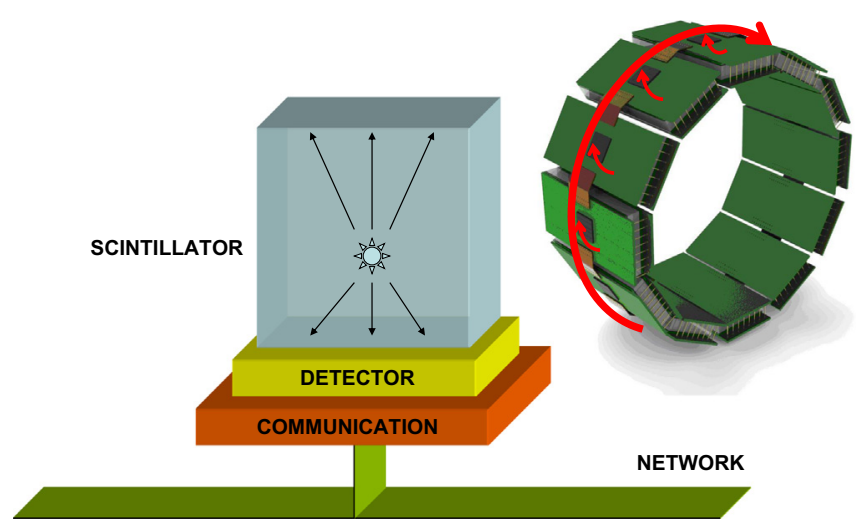

Fig. 1. SPADnet photonic module concept. Inset: PET ring assembly of coincidence data network.

Diode)-based sensor devices, featuring TSV (Through Silicon Via) connections to their backside (replacing conventional wire bonding), are tightly abutted on a single PCB to form a so-called sensor tile. The latter is interfaced to an FPGA-based PCB on its back, composing, together with the scintillator on top of the sensor tile, a photonic module, as shown in Fig. 1. The latter acts as an autonomous sensing and computing unit, individually detecting gamma photons as well as thermal and Compton events, being able to determine basic information for each scintillation event, such as exact time of arrival, position and energy.

\subsection{Networking}

The photonic modules themselves are interfaced in a ring-like fashion, with the coincidence detection taking place in a differed and distributed manner (to ensure scalability) within the ring into which each photonic module injects its data. The selected true coincidence events are then collected by a snooper module, from which they are transferred to an external reconstruction computer using Gigabit Ethernet connectivity. In the SPADnet approach, the first line of computing is therefore the ring itself [2].

Further, to enable monitoring the status of the neighboring nodes, two communication links acting in opposite directions to each other are used for inter-node communication. The SPADnet network is designed to operate in two stages. In the first stage, coincidence detection is performed, using small packets (30 bits) containing basic timing information. In the second stage the larger detected coincidence events or true events are transferred outside of the ring for further processing. Finally, the network architecture is implemented in such a way as to ensure scalability in the axial direction as well (multi-ring capability).

The network, implemented on a Spartan- $6^{\mathrm{TM}} /$ Virtex-6 $6^{\mathrm{TM}}$ backbone, reaches a data rate of over $2 \mathrm{Gbps}$ in module-to-module communication and is capable of processing up to 3.3 million events per second. Fig. 2 shows the actual implementation of the network and the concept of multi-ring communication in the inset.

\subsection{Sensor devices and sensor tile}

The sensor tile is composed of an array of individual, identical sensor devices manufactured in a standard $130 \mathrm{~nm}$, TSV enabled CMOS imaging process (STMicroelectronics). The first version of the sensor device comprises a $8 \times 16$ pixel array [1] based on CMOS small area SiPMs (mini-SiPMs) [3], and its micrograph is shown in Fig. 3. Each pixel is $570 \times 610 \mu \mathrm{m}^{2}$ in size and contains 4 digital mini-SiPMs, for a total of 720 SPADs, resulting in a pixel array fill-factor (FF) of $42.6 \%$. As highlighted in the micrograph, the

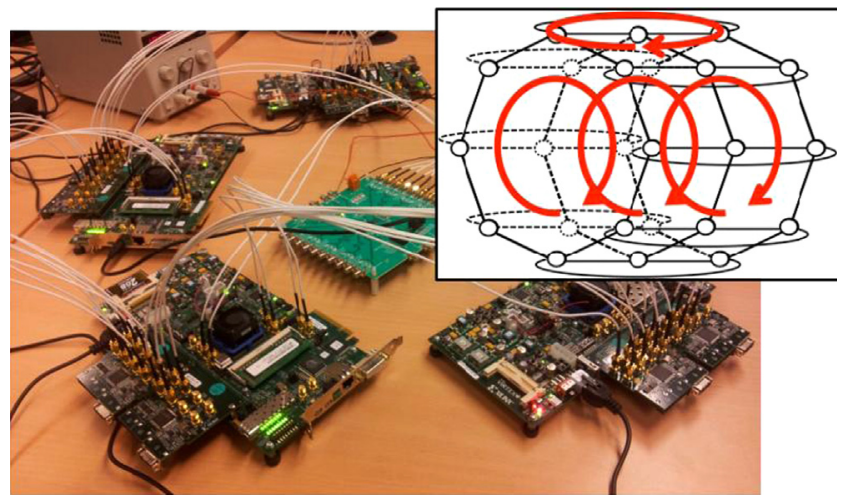

Fig. 2. Network experimental implementation. Inset: architecture of scalable multi-ring networks.

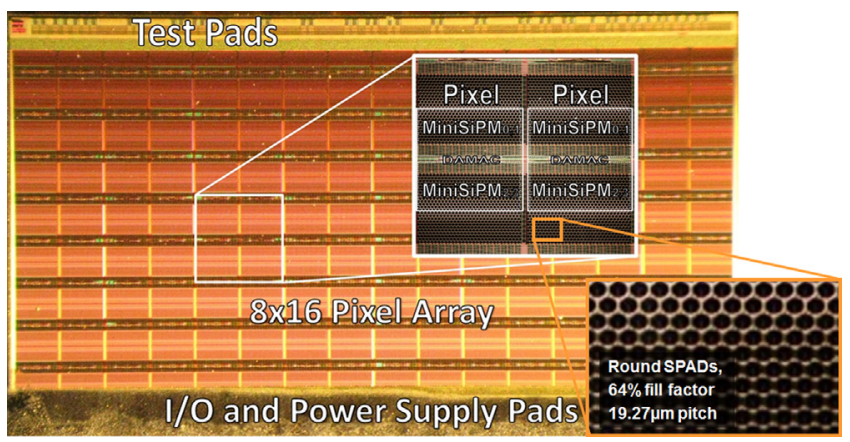

Fig. 3. Micrograph of the first SPADnet sensor. Insets: pixel and SPAD array in detail.

top pads are for testing purposes, and the full sensor operation has been verified using only the bottom pads. A production respin of the chip, therefore, could drop the top pads for an overall photonic module total FF of about $38 \%$.

The sensor operates on a $100 \mathrm{MHz}$ clock, which divides the sensor counting function in time bins. At each clock cycle, the sensor is able to generate its total counts value, which is then output in real-time. This stream of data, which represents the digitized photon flux, is also used by the on-chip discriminator. The function of the discriminator is to signal the external controller when a gamma event occurs, so that the sensor can be efficiently read out. Furthermore, for each gamma event the sensor also provides per-pixel energy and timestamping, obtained through 9-bit accumulators and 12-bit, 64 ps TDCs.

Preliminary testing of the sensor tile has been performed with a $3 \times 3 \times 5 \mathrm{~mm}^{3}$ LYSO scintillator at $20^{\circ} \mathrm{C}$. Fig. 4 shows the average image obtained from $511 \mathrm{keV}$ events, where the fine-grained pixel pitch results in a clear photon distribution. The real-time photon flux output is illustrated with three examples in Fig. 5, highlighting how it can be used to detect pile-ups. The main PET figures of merit, energy and timing resolution, were measured at $13.1 \%$ and 399 ps, respectively, as shown in Figs. 6 and 7. Finally, the discriminator was also evaluated, showing that it can completely hide dark-generated counts while at the same time being able to discriminate gamma energies as low as $40 \mathrm{keV}$.

\subsection{Scintillator and optical coupling}

In order to achieve good performance with PET modules based on digital SiPM technology we have to rethink and redesign the scintillator geometry and the optical elements coupled to it. The enhanced timing and spectral properties of the SPADnet sensor require a new approach from the module designer to exploit all its 


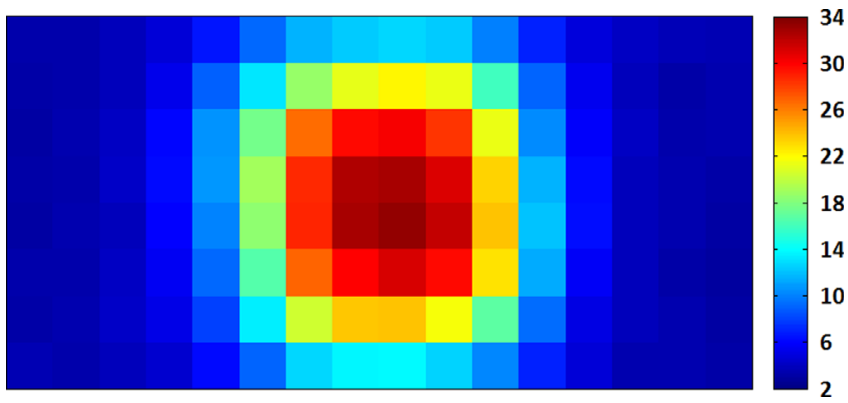

Fig. 4. Average image obtained with LYSO $3 \times 3 \times 5 \mathrm{~mm}^{3}$ crystal.

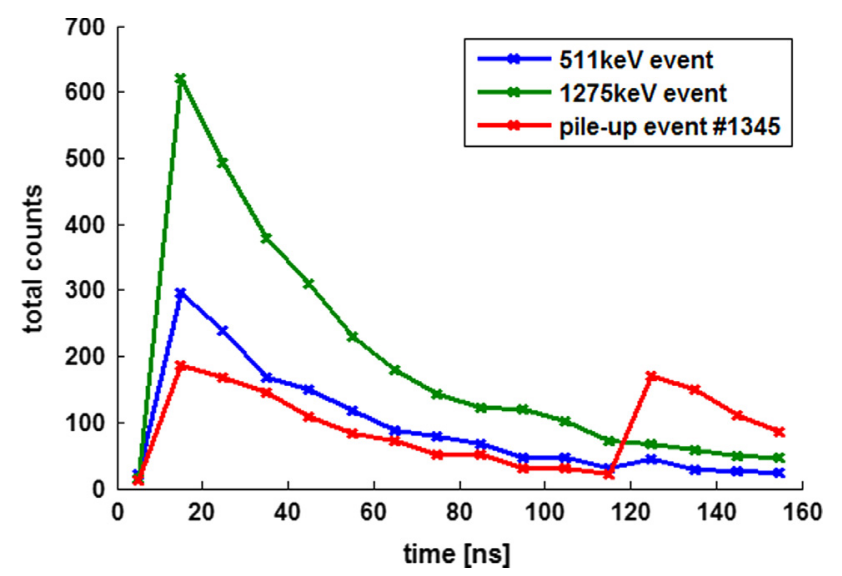

Fig. 5. Examples of the real-time energy output of the SPADnet sensor with three different types of events.

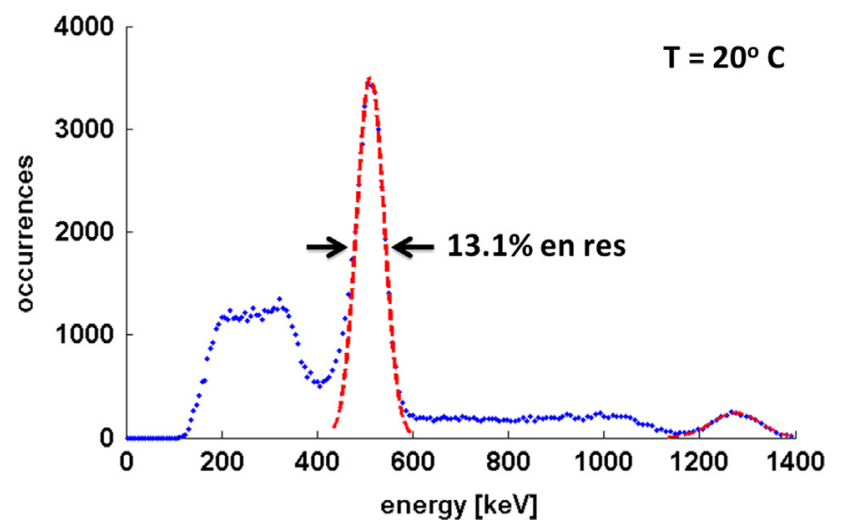

Fig. 6. Obtained energy resolution using a single Gaussian fit.

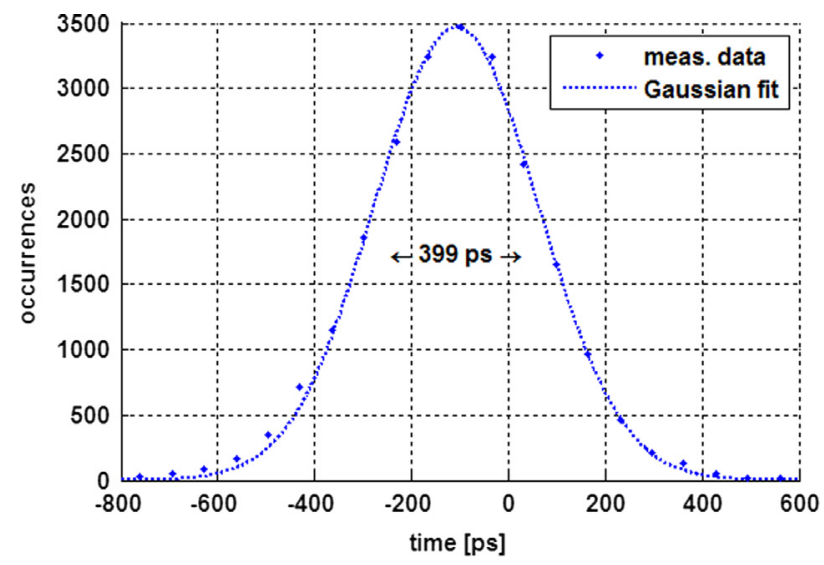

Fig. 7. Coincidence timing resolution obtained by combining 7 timestamps with the estimator from Ref. [4]. intrinsic benefits. Taking into account all these properties a Depthof-Interaction (DOI) capable PET module has been designed using single sided readout of a continuous LYSO:Ce scintillator crystal. This simple, basic construction supports our long term vision on a good price to performance ratio, scalable PET system.

Since this design problem is very unique we developed our own simulation environment that is capable of simulating the quantum-like behavior of light in photon starved environments. Our optical simulator called SCOPE [5] (Semi-Classic ObjectPreferred Environment) is based on a ZEMAX [6] and a suitable set of materials and geometric models for PET module simulation. The output of ZEMAX is fed into a specific MATLAB [7] code. SCOPE can be used as a stand-alone simulator or it can be connected to a detailed sensor simulator, also based on MATLAB, for refined analysis of the given construction.

Experimental validation of design principles and measurement results is always a mandatory step in such processes. For that purpose we developed a new measurement technique (Fig. 8) that makes it possible to excite our experimental PET modules in a single point at a time [8]. This is possible because we use UVexcitation instead of the conventional collimated $\gamma$-source to generate POIs. This capability is essential if we want to investigate DOI capabilities.

Using the tools mentioned above we have already tested new optical solutions (Fig. 9), such as the utilization of retro-reflectors on the $\gamma$-side of the module. This solution improved the spatial resolution. We also found that slanted side faces of the scintillator crystal are useful not only for increasing the sensitivity of a final PET system, but also for increasing photon extraction from the scintillator [9].

\subsection{Optical concentration and photonic module}

The efficient collection of light enables defining the energy of the gamma ray and the point of interaction within the scintillator. In order to recover the SPAD's fill factor loss, several optical concentrator (OC) geometries have been studied, taking into account the sensor design. The specificity of light concentration for scintillator crystals is due to the corresponding wide light angle distribution, unlike conventional imaging where the light is quite on axis. Due to the small SPAD size $(16.27 \mu \mathrm{m})$ and pitch $(19.27 \mu \mathrm{m})$, the technology used for the OC realization must be wafer level, and compatible with standard microelectronic process. Nanoimprint lithography enables making various shapes

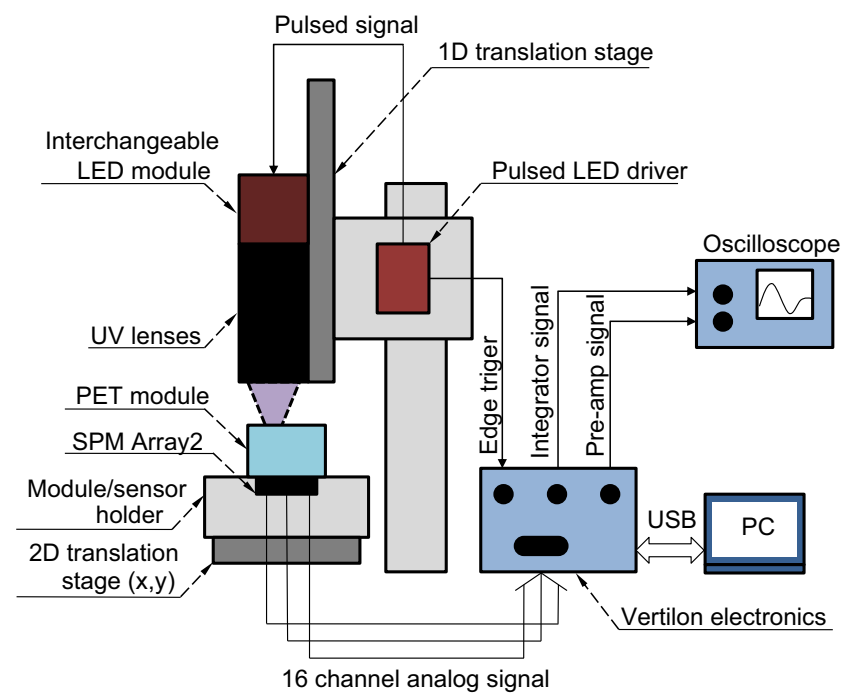

Fig. 8. Overview of the UV-excited measurement setup. 
(a)

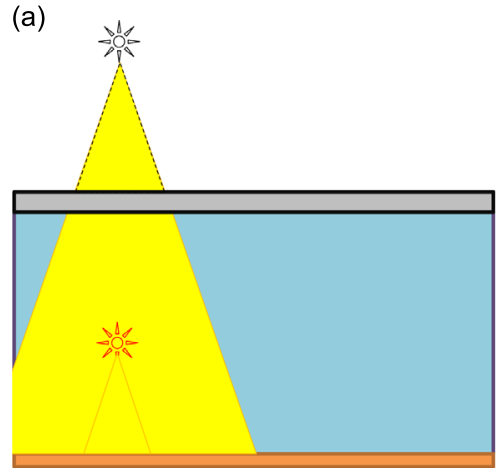

(c)
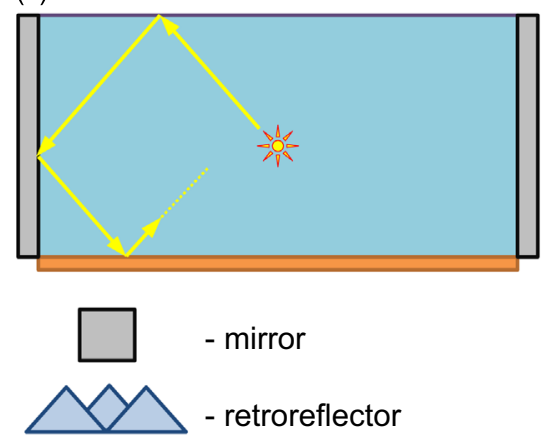

(b)

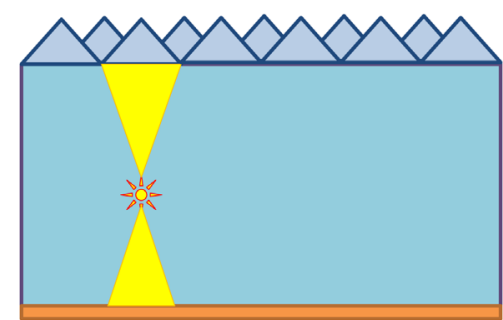

(d)

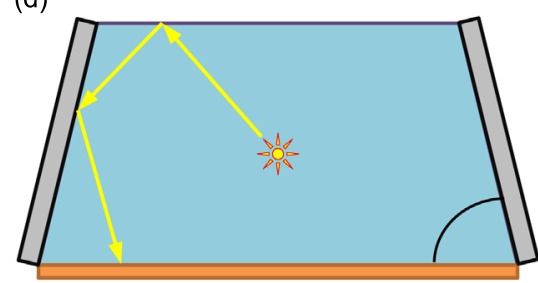

- scintillator

- sensor

Fig. 9. Detector modules with reflective $\gamma$-side surface (a), retro-reflective $\gamma$-side (b), reflective side faces (c) and slanted, mirrored side faces (d).

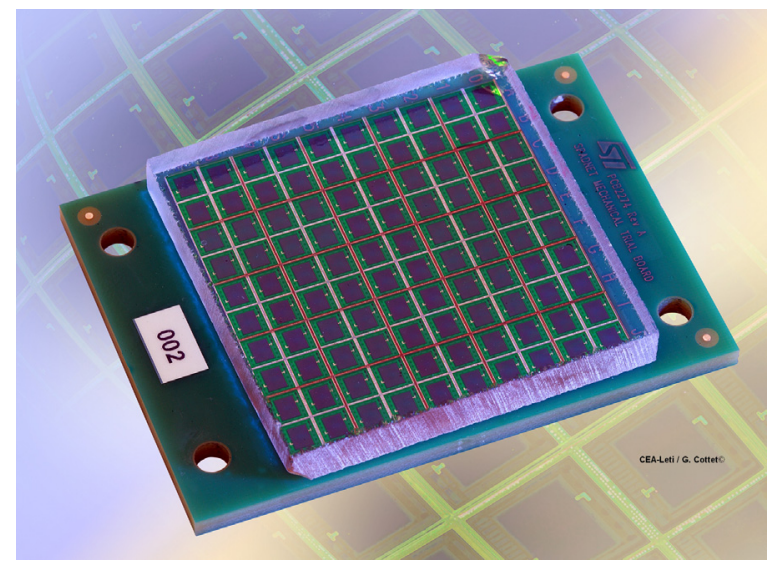

Fig. 10. Photonic module assembly with similar TSV chips and LYSO-like scintillator crystal slab.

using polymers. A large number of OC geometries have been studied and realized.

The photonic module will consist of an array of $5 \times 5$ tightly packed sensor tiles, whereas TSVs replace conventional wire bonding. A preliminary assembly of a LYSO slab with a dummy tile of 25 similar TSV chips on the PCB substrate with a minimum gap between chips has been demonstrated as can be seen in Fig. 10.

\section{Preliminary testing}

The design of a preclinical PET based on networked SPADnet photonic modules is well underway. A CAD drawing of the final system is presented in Fig. 11, showing a compact design that will be compatible with up to $9.4 \mathrm{~T}$ static fields, so as to enable

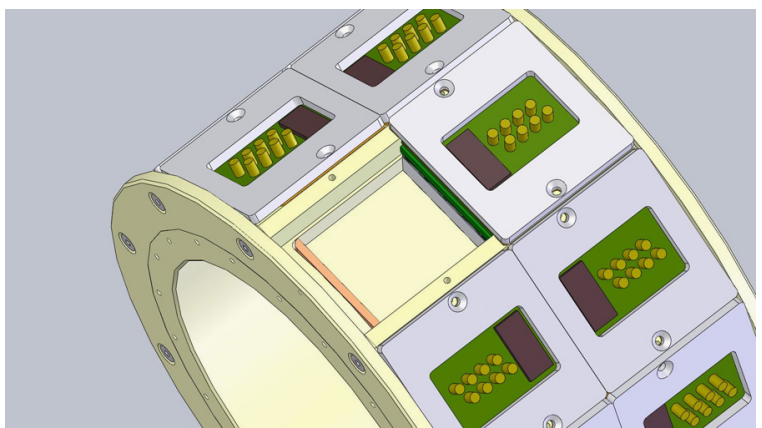

Fig. 11. CAD drawing of the final MRI compatible preclinical system assembly.

multi-modal PET imaging. We expect the first reconstructed PET images in the upcoming months.

\section{Conclusions}

We expect that the natively digital, networked SPADnet concept, where intelligence and ToF-enabling timestamping can be embedded on the sensor device, as well as very close to it using off-the-shelf programmable FPGA devices to enable real-time operation, will not only impact PET scalability but also performance robustness (reproducibility and parameter control) and ultimately cost. The sensor devices themselves can be manufactured in standard CMOS technology, enabling significant savings once produced in volume quantities. Another advantage is the capability of being compatible with magnetic resonance imaging (MRI), thus prompting advances in multimodal imaging and medical diagnostics as a whole. 


\section{Acknowledgments}

The research leading to these results has received funding from the European Union Seventh Framework Program under Grant Agreement No. 256984 - www.spadnet.eu. The authors are grateful to Xilinx, Inc., for its generous FPGA donations.

\section{References}

[1] L.H.C. Braga, L. Gasparini, L. Grant, R.K. Henderson, N. Massari, M. Perenzoni, D. Stoppa, R. Walker, An $8 \times 16$-pixel 92k SPAD time-resolved sensor with on-pixel $64 \mathrm{ps} 12 \mathrm{~b}$ TDC and $100 \mathrm{MS} / \mathrm{s}$ real-time energy histogramming in $0.13 \mu \mathrm{m}$ CIS technology for PET/MRI applications, in: Proceedings of the IEEE International Solid-State Circuits Conference, pp. 487-487, 2013.

[2] C. Veerappan, C. Bruschini and E. Charbon, Sensor network architecture for a fully digital and scalable SPAD based PET system, in: IEEE Nuclear. Science. Symposium. Conference. Record. (NSS/MIC), pp. 1115-1118, 2012.

[3] L.H.C. Braga, L. Pancheri, L. Gasparini, M. Perenzoni, R. Walker, R.K. Henderson, D. Stoppa, A CMOS mini-SiPM detector with in-pixel data compression for PET applications, in: IEEE Nuclear. Science. Symposium. Conference. Record. (NSS MIC), pp. 548-552, 2011.

[4] L.H.C. Braga, L. Gasparini, D. Stoppa, A time of arrival estimator based on multiple timestamps for digital PET detectors, in: IEEE Nuclear. Science. Symposium. Conference. Record. (NSS/MIC), pp. 1250-1252, 2012.

[5] B. Jatekos, G. Erdei, E. Lörincz, Simulation tool for optical design of PET detector modules including scintillator material and sensor array, in: Proceedings of the 2nd International Conference on Advancements in Nuclear Instrumentation Measurement Methods and their Applications (ANIMMA), 6-9 June 2011, pp. 1 6, http://dx.doi.org/10.1109/ANIMMA.2011.6172960.

[6] Radiant Zemax LLC, Zemax Design Tools [Online]. Available from: 〈http://www. radiantzemax.com/zemax 2013.

[7] MATLAB [Online]. Available from: 〈http://www.mathworks.com/products/ MATLAB/ $>$.

[8] B. Játékos, Z. Kolozsi, E. Lörincz, F. Ujhelyi, A. Barócsi, G. Erdei, Characterization of MRI-compatible PET detector modules by optical excitation of the scintillato material, in: Proceedings of SPIE 8439, Optical Sensing and Detection II, 84391R, April 23, 2012.

[9] B. Jatekos, A.O. Kettinger, E. Lorincz, F. Ujhelyi; G. Erdei, Evaluation of light extraction from PET detector modules using gamma equivalent UV excitation, in: IEEE Nuclear Science Symposium Conference Record (NSS/MIC) M21-6 pp. 3746-3750, 2012 